\title{
ANTICIPATED PLASMA WAVE MEASUREMENT ONBOARD EXOMARS 2020 SURFACE PLATFORM
}

\author{
I. Kolmasova*†, O. Santolik*†, and A. Skalsky ${ }^{\ddagger}$
}

\begin{abstract}
The ExoMars 2020 Surface Platform will conduct environmental and geophysical measurements with the aim of studying the Martian surface and subsurface environment at the landing location. The Surface Platform instrumentation will include the Wave Analyzer Module (WAM) as a European contribution to the Russian-led Martian ground electromagnetic tool (MAIGRET) instrument. The wave analyzer module will be dedicated to measurements of magnetic field fluctuations in the frequency band from $100 \mathrm{~Hz}$ to $20 \mathrm{kHz}$. The scientific questions which we plan to address by measurements of the WAM have never been answered by direct measurements of the fluctuating magnetic fields in the appropriate range of frequencies directly on the surface of the planet. The immediate questions related to these targets are: 1 . Can we observe electromagnetic radiation from electric discharges in the Martian dust storms? 2. Can we observe electromagnetic radiation propagating from the interplanetary space down to the surface of the planet?
\end{abstract}

\section{Introduction}

Very low frequency (VLF) wave measurements might help to find the sources responsible for the generation of electromagnetic radiation observable on the Martian surface. This electromagnetic radiation can originate in electric discharges in Martian dust storms. Plasma waves can also originate in the interplanetary space, penetrate the Martian ionosphere, and propagate down to the surface. This paper presents a preliminary description of a wave analyzer which will be placed on the ExoMars 2020 Surface platform in order to measure fluctuations of the magnetic field in the frequency range from several Hertz to $20 \mathrm{kHz}$ for the first time directly on the surface of the planet. In the second section we present an overview of past and current plasma wave measurements conducted from

\footnotetext{
* Institute of Atmospheric Physics, CAS, Prague, Czechia

$\dagger$ Faculty of Mathematics and Physics, Charles University, Prague, Czechia

$\ddagger$ Institute of Space Research, RAS, Moscow, Russia
} 
Martian orbit and calculations determining the conditions under which the wave penetration through the Martian ionosphere might occur. In the third section we discuss experimental and modeling results concerning the hypothesis of the existence of Martian lightning discharges. In the sections 4 and 5 we introduce the ExoMars Mission and the WAM instrument.

\section{Plasma wave measurements on Mars}

The first VLF plasma wave measurement from Martian orbit was conducted by the Plasma Wave System (PWS) onboard the Soviet PHOBOS 2 spacecraft. It was launched in July 1988 and entered Martian orbit in January 1989. It operated nominally during the cruise and orbital insertion phases, and stopped working in March 1989 because of a malfunction in the on-board computer. The instrument PWS recorded spectra of waves using an electric dipole made of two spheres $(10 \mathrm{~cm}$ in diameter) which were separated by a distance of $1.45 \mathrm{~m}$, and a filter bank consisting of 25 filters covering the frequency range from DC to $150 \mathrm{kHz}$. Electromagnetic whistler mode waves with frequencies below the local electron cyclotron frequency were recorded within the planetosphere [Grard et al., 1991]. The Mars-96 Orbiter spacecraft carrying a plasma wave complex named ELISMA was destroyed during its unsuccessful launch attempt in 1996. In the preparations for this mission several important theoretical studies were published [e.g., Melnik and Parrot, 1998; 1999]. The Japanese Planet-B Mars Orbiter also carrying a plasma waves and sounder instrument was launched in 1998, but the insertion into the Martian orbit failed because of the unsuccessful Earth swing-by maneuver.

The Mars Atmosphere and Volatile EvolutioN (MAVEN) mission launched in 2013 is equipped with a Langmuir Probe and the Waves (LPW) instrument which is designed to measure the power spectral density of waves (DC-2 MHz) observed in the Martian ionosphere and magnetosphere. The LPW instrument uses two cylindrical sensors $(40 \mathrm{~cm}$ long, $0.635 \mathrm{~cm}$ diameter) with pre-amplifiers. The sensors are mounted on two 7 meter long stacer booms. The LPW instrument also measures the electron density and temperature in the ionosphere of Mars [Andersson et al., 2015]. Harada et al. [2016] report the LPW observation of narrowband whistler-mode electromagnetic waves at frequencies between the lower hybrid and local electron cyclotron frequencies in the Martian induced magnetosphere on both open and closed field lines. Some spectra recorded on closed field lines contain discrete rising tone elements and two bands above and below half the electron cyclotron frequency. The authors hypothesize that these whistler mode waves could be generated by cyclotron resonance with anisotropic electrons. Ruhunusiri et al. [2016] report the LPW observation of large amplitude VLF left-hand polarized waves propagating obliquely to the ambient magnetic field in the upstream region of Mars.

Up to now there has not been any VLF plasma wave measurement conducted from the surface of Mars. With such measurement we can investigate if electromagnetic radiation originating in the interplanetary space can propagate down to the surface of the planet. Mars lacks a strong intrinsic planetary magnetic field, and the solar wind interacts directly with the Martian ionosphere. Melnik and Parrot [1999] characterize conditions under which these waves penetrate the Martian ionosphere and can be observed on the surface. 
They showed that considering only a low magnetic field induced by the solar wind, the wave penetration through the Martian ionosphere would not be possible. According to their calculations waves can propagate through the ionosphere only if the local crustal magnetic field is strong enough. Such a high value of magnetic field was measured by the Mars Global Surveyor probe above patches of highly magnetized crust on the Martian surface. On average the Mars crust is 10 times more intensely magnetized than that of Earth [Connerney et al., 2004]. Melnik and Parrot [1999] concluded that waves below $4 \mathrm{kHz}$ can penetrate the Martian ionosphere above these specific magnetized regions in the night side during low solar activity.

\section{Possibility of atmospheric discharges on Mars}

Based on simulations and laboratory experiments, and in an analogy with terrestrial volcanic and dust discharges, electric discharges are expected to develop in Martian dust storms. Dust grains are expected to get charged by collisions. Knowing the properties of the Martian dust grains Melnik and Parrot [1998] calculated an evolution of charge density and an electrostatic potential for two different dust storm configurations (a wind vortex and a horizontal wind encountering a small hill). Numerical results indicate that it is possible to reach an electrical breakdown in the low-pressure carbon dioxide Martian atmosphere under specific particle density, particle composition and sufficiently high wind velocity. The authors showed that if small and large dust particles collide in a vortex or a horizontal wind, then the dust particles get charged. The charge polarity is dependent on the dimensions of the grains. The charged particles are then vertically separated due to the gravitational force and as a result an electric field between oppositely charged dust particle layers is established. If the strength of the field exceeds the electric breakdown for low-pressure atmosphere $(5-20 \mathrm{kV} / \mathrm{m})$ then a discharge can occur. The authors also showed that the time to reach the breakdown increases with decreases in charge density, weaker charging of individual grains and decreasing wind velocity. If the recombination of charges is too high it is impossible to achieve the breakdown.

Laboratory experiments confirm the existence of visible electrical discharges in clouds of Martian regolith simulant in a low-pressure carbon dioxide atmosphere. These discharges were achieved for specific composition of dust particle sizes $(51 \% 250-1000 \mu \mathrm{m}, 24 \% 150$ $249 \mu \mathrm{m}$ and $25 \%<150 \mu \mathrm{m})$ in a range of simulated wind speeds between 1.4 and $5.2 \mathrm{~m} / \mathrm{s}$ [Kraus et al., 2003].

Farrell et al. [2006] observed an ultra-low frequency magnetic radiation generated by terrestrial dust devils. The authors confirmed also by simulations that grains in a dust devil become charged by collisions and that a distribution of fluctuating charges in a convective vortex leads to measurable magnetic fields. Martian dust devils and dust storms are more frequent, stronger, and larger than the ones on Earth. Renno et al. [2003] presented theoretical arguments supporting a hypothesis that Martian dust storms produce non-thermal wideband radiation detectable from Earth.

Lightning discharges are also frequently observed in many types of volcanic eruptions. Small scale discharges (with the length of lightning channels on the order of 10-100 m) 
occur directly above the vent during the explosive period of the eruption. Large scale discharges were observed in the plumes of volcanic ash [Behnke et al., 2013]. The plumes can be up to $10 \mathrm{~km}$ long in the later stages of volcanic eruptions. An impulsive current flowing in a lightning channel within a long ash plume can generate electromagnetic signals in the VLF range. It was found that electromagnetic signals originating in volcanic lightning discharges produced during the eruption of Mount Okmok volcano (Aleutian Islands) in 2008 traveled along the connecting magnetic field line to the opposite hemisphere and were recorded as whistlers at the conjugated point in Dunedin, New Zealand [Antel et al., 2014].

Although these results may indicate the existence of atmospheric lightning discharges on Mars, currently there is no optical evidence of the existence of Martian lightning discharges. Impulsive radio signals originating in electric discharges from the Martian dust storms have also never been detected from space. Gurnett et al. [2010] reported an unsuccessful search for electromagnetic impulsive radio signals penetrating through Martian ionosphere using the MARSIS instrument onboard Mars Express spacecraft. The search covered altitudes from 275 to $1400 \mathrm{~km}$ and frequencies from 4.0 to $5.5 \mathrm{MHz}$. Radio observations of Mars from Earth showed anomalous strong microwave emissions in regions of enhanced dust activity [Renno et al., 2003; Ruf et al., 2009]. Ruf et al. [2009] found that the spectrum of the observed radiation has significant peaks around predicted values of three lowest modes of the Martian Schumann Resonance. The authors concluded that observed non-thermal radiation was probably caused by electric discharges in a Martian dust storm.

\section{ExoMars mission}

The main goal of the ESA ExoMars 2016+2020 spacecraft missions is to establish if life existed on Mars. The ExoMars 2016 mission consisted of the Trace Gas Orbiter (TGO) and an Entry, Descent and landing demonstrator Module (EDM), known as Schiaparelli. The spacecraft was launched in March 2016. The orbiter was inserted in the Martian orbit in October 2016. It is dedicated to detect and study atmospheric trace gases, such as methane. The Schiaparelli module unfortunately crashed during the descent phase. The ExoMars 2020 mission will have two science elements that will land on Mars. It is composed of a rover and a surface platform (SP). The rover will carry a drill and a suite of instruments to conduct exobiological and geochemistry research. The surface platform will conduct environmental and geophysical measurements of the surface and subsurface environment at the landing location. The other science priorities for the surface platform are long-term climate monitoring and atmospheric investigations. The suite of SP instruments includes the radio-science experiment LARA, the Habitability, brine irradiation and temperature package HABIT, the METEO M meteorological package, the MAIGRET magnetometer, a set of cameras to characterize the landing site environment, the infra-red Fourier spectrometer FAST to study the atmosphere, the active neutron spectrometer and dosimeter ADRON-EM, the Multi-channel Diode-Laser Spectrometer for atmospheric investigations M-DLS, the Radio thermometer for soil temperatures PAT-M, the Dust suite, the seismometer SEM, 
and the Gas chromatography-mass spectrometry for atmospheric analysis MGAP [http: //exploration.esa.int/mars/56933-exomars-2020-surface-platform/]. The data relay services will be provided by the Trace Gas Orbiter already launched as a part of the 2016 ExoMars mission.

\section{$5 \quad$ Wave Analyzer Module (WAM)}

The Wave Analyzer Module is an essential part of the MAIGRET instrument dedicated to the monitoring of Martian electromagnetic environment on the surface of the planet. Up to now such direct measurements of the fluctuating magnetic fields have never been conducted. The WAM is accommodated inside the MAIGRET box of electronics and will perform digitization and onboard processing of the signal from a magnetic field search coil. The basic WAM observing periods will be related to the intervals between data relays through the ExoMars 2016 TGO spacecraft. The WAM will be able produce a continuous data stream of survey data, completed with short intervals of selected high resolution burst mode data, transmitted for the data relay at the end of each observational period. The survey data will be composed of averaged spectral data from search coil magnetometer measurements which will be computed and analyzed onboard. The time separation between individual spectra will be chosen according to the available data rate from $1 \mathrm{~s}$ to several seconds. The survey data will provide us with an overview of the overall wave activity. This data set will be used to investigate the higher frequency components of interactions of interplanetary plasma medium with the Martian ionosphere and with the Martian magnetic anomalies on the surface. It will also characterize the ionosphere-atmosphere-lithosphere interactions on Mars related to space weather effects. The burst mode data stream will consist of discrete snapshots of waveform data or their high resolution spectra. The time resolution for the burst mode waveforms will be 20 $\mu \mathrm{s}$. To monitor fast signals a triggering algorithm based on the waveform data will be implemented. The maximum will be found over a series of samples and compared with a predefined threshold. A trigger will be generated if the calculated maximum exceeds the threshold. Waveform snapshots will be recorded around the trigger time. All parameters of the triggering procedure will by adjustable by a telecommand. The burst will be used to investigate details of above mentioned processes and also to search for possible electromagnetic emissions of atmospheric origin related to dust storms, and for possible wave activity originated in electrical discharges.

\section{Conclusions}

The final landing site of the ExoMars 2020 mission will be selected according to its main objective, which is a search for life. The places with highly magnetized crust patches are unfortunately outside two preselected landing sites. Landing sites Oxia Planum and Mawrth Vallis were preselected in March 2017 because of the highest probability of a possible historical presence of liquid water at these sites. In spite of this fact we hope 
that using the WAM instrument placed directly on the Martian surface we can address the following questions:

a) Which frequencies and plasma wave modes can penetrate down to the surface of Mars?

b) What are the conditions under which we observe the penetration of electromagnetic radiation from interplanetary space down to the surface of Mars?

c) What state of the Martian ionosphere is the most favorable for the penetration to happen?

d) Are the waveforms of the electromagnetic radiation from Martian discharges similar to the waveforms radiated from terrestrial lightning?

e) Which processes lead to initial breakdown of Martian discharges and how are these processes reflected in the detectable electromagnetic radiation?

f) Which special meteorological conditions lead to initiation of Martian discharges?

g) Are the Schumann resonances detectable on Mars?

Acknowledgments. This work was supported by the Praemium Academiae award of the CAS. The Editors thank two anonymous reviewers for their help in evaluating this paper.

\section{References}

Andersson, L, R. E. Ergun, G. T. Delory, A. Eriksson, J. Westfall, H. Reed, J. McCauly, D. Summers, and D. Meyers, The Langmuir Probe and Waves (LPW) instrument for MAVEN, Space Sci. Rev., 195, 173-198, 2015.

Antel, C., A. B. Collier, J. Lichtenberger, and C. J. Rodger, Investigating Dunedin whistlers using volcanic lightning, Geophys. Res. Lett., 41, 4420-4426, 2014.

Behnke, S. A, R. J. Thomas, S. R. McNutt, D. J. Schneider, P. R. Krehbiel, W. Rison, and H. E. Edens, Observations of volcanic lightning during the 2009 eruption of Redoubt Volcano, J. Volcanology and Geothermal Res., 259, 214-234, 2013.

Connerney, J.E.P., M. H. Acuña, N.F. Ness, T. Spohn, and G. Schubert, Mars crustal magnetism, Space Sci. Rev., 111, 1-32, 2004.

Farrell, W. M., J. R. Marshall, S. A. Cummer, G. T. Delory, and M. D. Desch, A model of the ULF magnetic and electric field generated from a dust devil, J. Geophys. Res., 111, E11004, 2006.

Grard, R., C. Nairn, A. Pedersen, S. Klimov, S. Savin, A. Skalsky, and J. G. Trotignon, Plasma and waves around Mars, Planet. Space Sci., 39, 89-91, 1991.

Gurnett, D. A., D. D. Morgan, L. J. Granroth, B. A. Cantor, W. M. Farrell, and J. R. Espley, Non-detection of impulsive radio signals from lightning in Martian dust storms 
using the radar receiver on the Mars Express spacecraft, Geophys. Res. Lett., 37, L17802, 2010.

Harada, Y., L. Andersson, C. M. Fowler, D. L. Mitchell, J. S. Halekas, C. Mazelle, J. Espley, G. A. DiBraccio, J. P. McFadden, D. A. Brain, S. Xu, S. Ruhunusiri, D. E. Larson, R. J. Lillis, T. Hara, R. Livi, and B. M. Jakosky, MAVEN observations of electron-induced whistler mode waves in the Martian magnetosphere, J. Geophys. Res., 121, 10, 9717-9731, 2016.

Krauss, C.E., M. Horanyi, and S. Robertson, Experimental evidence for electrostatic discharging of dust near the surface of Mars, New J. of Phys., 5, 70.1-70.9, 2003.

Melnik, O., and M. Parrot, Electrostatic discharges in Martian duststorms, J. Geophys. Res., 103, 12705-12714, 1998.

Melnik, O., and M. Parrot, Propagation of electromagnetic waves through Martian ionosphere, J. Geophys. Res., 104, 29107-29117, 1999.

Renno, N. O., A.S. Wong, S. K. Atreya, I. de Pater, and M. Roos-Serote, Electrical discharges and broadband radio emission by Martian dust devils and dust storms, Geophys. Res. Lett., 30, id.2140, 2003.

Ruf, C., N. O. Renno, J.F. Kok, E. Bandelier, M. J. Sander, S. Gross, L. Skjerve, and B. Cantor, Emission of non-thermal microwave radiation by a Martian dust storm, Geophys. Res. Lett., 36, L13202, 2009.

Ruhunusiri, S., et al. (12 co-authors), MAVEN observation of an obliquely propagating low-frequency wave upstream of Mars, J. Geophys. Res., 121, 2374-2389, 2016. 
